\title{
Urinary tissue inhibitor of metalloproteinase-2 and insulin-like growth factor-binding protein 7 as biomarkers of patients with established acute kidney injury
}

\author{
Won Yong Cho*, Sung Yoon Lim, Ji Hyun Yang, Se Won Oh, Myung-Gyu Kim, and Sang-Kyung Jo
}

Department of Internal Medicine, Korea University College of Medicine, Seoul, Korea

Received: July 17, 2018 Revised : December 8, 2018 Accepted: January 30, 2019

\section{Correspondence to} Myung-Gyu Kim, M.D. Department of Internal Medicine, Korea University Anam Hospital, Korea University College of Medicine, 73 Inchon-ro, Seongbuk-gu, Seoul 02841, Korea

Tel: +82-2-920-5909

Fax: +82-2-927-5344

E-mail:gyu219@hanmail.net

*These authors contributed equally to this work.
Background/Aims: Urinary tissue inhibitor of metalloproteinase-2 (TIMP-2) and insulin-like growth factor-binding protein 7 (IGFBP7) have been recently discovered and validated as sensitive biomarkers that can predict stage 2 or 3 acute kidney injury (AKI) development in high-risk patients. We aimed to assess whether these biomarkers could predict adverse outcomes and renal recovery in established AKI patients.

Methods: This was a single-center study prospectively enrolling 124 patients diagnosed with AKI. TIMP-2, IGFBP7, neutrophil gelatinase-associated lipocalin (NGAL), and kidney injury molecule 1 (KIM-1) levels were measured at the time of diagnosis and the predictive performance of short-term outcomes and renal recovery was assessed.

Results: Patients were divided into 4 quartiles according to the initial urinary TIMP-2/IGFBP7 levels. Stage 3 AKI (odds ratio [OR], 17.86), classified by the Kidney Disease Improving Global Outcomes (KDIGO), as well as the third and fourth quartiles of TIMP-2/IGFBP7 (OR, 5.75 and 44.98, respectively), were found to be independent predictors of renal replacement therapy at the time of AKI diagnosis. In addition, KDIGO stage $3 \mathrm{AKI}(\mathrm{OR}, 2.468)$ or the third of fourth quartiles of urinary TIMP-2/IGFBP7 (OR, 1.896 and 3.622, respectively) were also found to be useful in predicting nonrecovery of renal function. In a separate analysis of patients with renal recovery at discharge, initial urinary TIMP-2/IGFBP7 or urinary $\mathrm{IGFBP}_{7}$ at discharge could also predict new-onset or progressive chronic kidney disease (CKD).

Conclusions: In AKI patients, urine TIMP-2/IGFBP7 could serve as a biomarker for predicting adverse outcomes, renal recovery, or the development and progression of CKD.

Keywords: Acute kidney injury; Biomarker; Tissue inhibitor metalloproteinase-2; Insulin-like growth factor-binding protein 7 


\section{INTRODUCTION}

Acute kidney injury (AKI) is associated with high mortality and adverse long-term outcomes [1,2]. It can also lead to the development of chronic kidney disease (CKD), worsen pre-existing CKD, and accelerate CKD progression to end-stage renal disease (ESRD) $[3,4]$. Although epidemiological studies have demonstrated that survivors of AKI episodes who require temporary dialysis are at an extremely high-risk for progressive CKD, there is no way to accurately predict renal recovery [5].

For several decades, substantial progress in the discovery and validation of novel biomarkers for early detection of AKI has been made. Recently, NephroCheck, which can detect urinary tissue inhibitor of metalloproteinase-2 (TIMP-2) and insulin-like growth factor-binding protein 7 ( $\left.\mathrm{IGFBP}_{7}\right)$ simultaneously, was approved for predicting the development of moderate-to-severe AKI (Kidney Disease Improving Global Outcomes [KDIGO] stage 2 or 3 ) within 12 hours in high-risk patients [6]. However, most of the previous studies on biomarkers have tested their ability to predict the development of AKI and only a few studies have addressed their performance as a prognostic marker for renal recovery or to assess the need for renal replacement therapy (RRT). Accurate prediction of recovery or progression to CKD following AKI might help clinicians recognize whether or not a patient is likely to recover and individualize long-term treatment plans accordingly.

TIMP-2 and IGFBP7 are Gi cell cycle arrest markers that are upregulated early after AKI onset and have also been proposed to have a renoprotective role. However, the observation that a prolonged cell cycle arrest results in maladaptive repair led us to hypothesize that a prolonged upregulation of these markers might represent the development or progression of CKD following AKI $[7,8]$.

This study measured urinary neutrophil gelatinase-associated lipocalin (NGAL), kidney injury molecule 1 (KIM-1), TIMP-2, and IGFBP7 when AKI was diagnosed and assessed their performance in predicting dialysis requirement and functional recovery. In addition, we also measured these biomarkers at the time of discharge in surviving patients without the need for RRT and tested the ability of these biomarkers in predicting new-onset or progressive CKD.

\section{METHODS}

\section{Subjects}

This study was a prospective cohort study of hospitalized patients with established AKI at the tertiary teaching hospital, Korea University Anam Hospital, in Seoul, Korea, conducted between June 2015 and July 2016. The study was approved by the Institutional Review Board of the Korea University Anam Hospital (IRB no.: 2012ANo290). Patients who were admitted or referred to the Nephrology Department because of a clinical diagnosis of AKI with varying degrees of renal dysfunction and with different etiologies were enrolled. AKI was defined and staged according to KDIGO criteria as follows: stage 1 , serum creatinine $\geq 0.3 \mathrm{mg} / \mathrm{dL}$ ( 48 hours) or 1.5-fold increase from baseline ( 7 days); stage 2 , the serum creatinine increased from 2.0 to 2.9 times the baseline; stage 3, serum creatinine increased more than 3.0 times the baseline or serum creatinine $\geq 4.0 \mathrm{mg} / \mathrm{dL}$ or the initiation of renal replacement therapy. Only those patients with known baseline serum creatinine levels within 6 months of enrollment were analyzed. The study was approved by the Institutional Review Board of this center and written informed consent was obtained from each patient or legal guardians before enrollment. Patients under 18 years of age, those undergoing chronic dialysis, those with stage 4 or more advanced CKD, or those with a history of kidney transplantation were excluded from the study. Data regarding patient demographics, comorbid conditions, baseline renal function, and 3-month follow-up renal function after hospital discharge in survivors were recorded.

\section{Samples and measurement of biomarkers}

Urine samples were collected at the time of enrollment from all patients and immediately before hospital discharge from surviving patients who did not require permanent dialysis. Supernatants were frozen and stored at $-70^{\circ} \mathrm{C}$. TIMP-2, IGFBP 7 , and NGAL levels were measured by enzyme-linked immunosorbent assay (ELISA) according to the manufacturer's instructions (TIMP-2, Abcam, Cambridge, UK; IGFBP 7 , Biorbyt, Cambridge, UK; NGAL, BioPorto, Gentofte, Denmark; and KIM-1, LSBio, Seattle, WA, USA). The amount of urine NGAL and KIM-1 excretion was expressed as $\mathrm{ng} / \mathrm{mL}$ for comparison and normalized to the urine creatinine concen- 
Table 1. Patient basal characteristics according to urine TIMP-2/IGFBP 7 excretion quartiles

\begin{tabular}{|c|c|c|c|c|c|c|}
\hline Characteristic & Total $(n=124)$ & $\begin{array}{l}\text { First }(\mathrm{n}=31) \\
\quad(<1.10)\end{array}$ & $\begin{array}{c}\text { Second }(\mathrm{n}=31) \\
\left(1.10-1.5^{8}\right)\end{array}$ & $\begin{array}{l}\text { Third }(\mathrm{n}=31) \\
(1.59-2.11)\end{array}$ & $\begin{array}{l}\text { Fourth }(\mathrm{n}=31) \\
\qquad(2.12 \leq)\end{array}$ & $p$ value \\
\hline Age, yr & $71.05 \pm 14.32$ & $73.90 \pm 10.73$ & $71.16 \pm 18.09$ & $71.48 \pm 13.48$ & $67.65 \pm 14.02$ & 0.196 \\
\hline Male sex & $62(50)$ & $14(45 \cdot 2)$ & $16(51.6)$ & $14(45 \cdot 2)$ & $18(58.1)$ & 0.701 \\
\hline $\mathrm{DM}$ & $5^{8}(46.8)$ & $17(54 \cdot 4)$ & $16(51.6)$ & $9(29)$ & $16(27.6)$ & 0.150 \\
\hline HTN & $87(70.2)$ & $24(77.4)$ & $21(67.7)$ & $21(67.7)$ & $21(67.7)$ & 0.792 \\
\hline CKD & $61(49.2)$ & $21(67.7)$ & $12(38.7)$ & $17(54.8)$ & $11(35 \cdot 5)$ & 0.039 \\
\hline Severity of AKI & & & & & & 0.317 \\
\hline KDIGO stage 1 & $45(36.3)$ & $16(51.6)$ & $12(38.7)$ & $9(29.0)$ & $8(25.8)$ & \\
\hline KDIGO stage 2 & $37(29.8)$ & $9(25.8)$ & $7(9 \cdot 3)$ & $12(38.7)$ & $10(32.3)$ & \\
\hline KDIGO stage 3 & $42(33 \cdot 9)$ & $7(22.6)$ & $12(38.7)$ & $10(32.3)$ & $13(41.9)$ & \\
\hline Baseline serum $\mathrm{Cr}, \mathrm{mg} / \mathrm{dL}$ & $1.21 \pm 0.46$ & $1.44 \pm 0.66$ & $1.22 \pm 0.41$ & $1.08 \pm 0.29$ & $1.08 \pm 0.41$ & 0.994 \\
\hline Admission serum $\mathrm{Cr}, \mathrm{mg} / \mathrm{dL}$ & $3.00 \pm 1.95$ & $2.84 \pm 1.25$ & $2.91 \pm 1.51$ & $2.94 \pm 1.84$ & $3.32 \pm 2.86$ & 0.994 \\
\hline Baseline eGFR, $\mathrm{mL} / \mathrm{min} / 1.73 \mathrm{~m}^{2}$ & $61.14 \pm 25.53$ & $59.17 \pm 32.91$ & $62.22 \pm 26.97$ & $58.64 \pm 17.32$ & $64.52 \pm 22.68$ & 0.870 \\
\hline Urine TIMP-2 at discharge, $\mathrm{ng} / \mathrm{mL}$ & $3.40 \pm 5.97$ & $1.65 \pm 1.90$ & $2.47 \pm 2.70$ & $2.84 \pm 2.63$ & $7.27 \pm 11.24$ & 0.008 \\
\hline Urine $\mathrm{IGFBP}_{7}$ at discharge, $\mathrm{ng} / \mathrm{mL}$ & $13.29 \pm 14.45$ & $14.91 \pm 25.21$ & $12.18 \pm 7.76$ & $11.40 \pm 5.22$ & $14.65 \pm 13.06$ & 0.824 \\
\hline Causes of AKI & & & & & & 0.760 \\
\hline Postrenal & $4(3.2)$ & $2(6.5)$ & $2(6.5)$ & 0 & o & \\
\hline Prerenal & $56(45 \cdot 2)$ & $14(45 \cdot 2)$ & $16(51.6)$ & $15(48.4)$ & $11(35 \cdot 5)$ & \\
\hline Intrinsic & $59(47.6)$ & $13(41.9)$ & $13(41.9)$ & $15(48.4)$ & $18(58.1)$ & \\
\hline Unknown & $5(4.0)$ & $2(6.5)$ & 0 & $1(3.2)$ & $2(6.5)$ & \\
\hline
\end{tabular}

Values are presented as mean $\pm \mathrm{SD}$ or number $(\%)$.

TIMP-2, tissue inhibitor of metalloproteinase-2; $\mathrm{IGFBP}_{7}$, insulin-like growth factor-binding protein 7; DM, diabetes mellitus; HTN, hypertension; CKD, chronic kidney disease; AKI, acute kidney injury; KDIGO, Kidney Disease Improving Global Outcomes; $\mathrm{Cr}$, creatinine; eGFR, estimate glomerular filtration rate.

tration (ng/mg creatinine).

\section{Clinical outcome}

The primary outcome was the initiation of dialysis or in-hospital mortality. The secondary outcome was the recovery of renal function at the time of discharge, defined as the return of serum creatinine to $25 \%$ of the baseline value. Development of new-onset CKD or progression of CKD was determined at three months after hospital discharge and defined as new-onset proteinuria or decline in the estimate glomerular filtration rate (eGFR) by $25 \%$ or more compared with the baseline eGFR. eGFR was estimated using an equation developed by the Chronic Kidney Disease Epidemiology Collaboration [9].

\section{Statistics}

All statistical analyses were performed using SPSS version 19.0 (IBM Co., Armonk, NY, USA). The results are expressed as the mean $\pm \mathrm{SD}$ for normally distributed data, or the median (interquartile range) for non-normally distributed data. Comparison between multiple groups was analyzed by an analysis of variance or the Kruskal-Wallis test in case of numerical data, and the chi-square test or Fisher's exact test in case of categorical data. Multiple comparisons were controlled by using the Bonferroni correction. Categorical variables were expressed as numbers with proportions. We conducted univariate and multivariate logistic regression analyses to assess the impact of different baseline characteristics at admission on the outcomes. We could select some significant factors in multivariate logistic regression by the backward selection based on likelihood ratio. To 
Table 2. Clinical outcomes according to urine TIMP-2/IGFBP 7 excretion quartiles

\begin{tabular}{|c|c|c|c|c|c|c|}
\hline Variable & Total $(\mathrm{n}=124)$ & $\begin{array}{c}\text { First }(\mathrm{n}=31) \\
(<1.10)\end{array}$ & $\begin{array}{c}\text { Second }(\mathrm{n}=31) \\
\quad(1.59-2.11)\end{array}$ & $\begin{array}{c}\text { Third }(\mathrm{n}=31) \\
(1.59-2.11)\end{array}$ & $\begin{array}{l}\text { Fourth }(\mathrm{n}=31) \\
\qquad(2.12 \leq)\end{array}$ & $p$ value \\
\hline In hospital mortality & $7(5.6)$ & o & $2(6.5)$ & $2(6.5)$ & $3(9.7)$ & 0.514 \\
\hline Renal replacement therapy & $16(12.9)$ & $1(3.2)$ & $2(6.5)$ & $4(12.9)$ & $9(29)$ & 0.002 \\
\hline Recovery & $90(72.6)$ & $29(93.5)$ & $22(71)$ & $22(71)$ & $17(54.8)$ & 0.008 \\
\hline Length of hospital stay, day & $12.67 \pm 7.48$ & $13.55 \pm 8.80$ & $10.84 \pm 6.17$ & $10.74 \pm 4.73$ & $15 \cdot 55 \pm 8.66$ & 0.087 \\
\hline
\end{tabular}

Values are presented as number (\%) or mean \pm SD.

TIMP-2, tissue inhibitor of metalloproteinase-2; IGFBP7, insulin-like growth factor-binding protein 7 .

Table 3. Univariate and multivariate analyses for predicting renal replacement therapy

\begin{tabular}{|c|c|c|c|c|}
\hline \multirow{2}{*}{ Variable } & \multicolumn{2}{|c|}{ Univariate analysis } & \multicolumn{2}{|c|}{ Multivariate analysis } \\
\hline & OR $(95 \% \mathrm{CI})$ & $p$ value & OR $(95 \% \mathrm{CI})$ & $p$ value \\
\hline Age & $2.28(0.18-28.71)$ & 0.523 & & \\
\hline Sex & $0.153(0.023-1.036)$ & 0.054 & $0.198(0.039-1.011)$ & 0.072 \\
\hline $\mathrm{DM}$ & $0.355(0.052-2.404)$ & 0.289 & & \\
\hline HTN & $1.991(0.532-7.449)$ & 0.305 & & \\
\hline CKD & $8.257(0.868-78.532)$ & 0.066 & & \\
\hline \multicolumn{5}{|c|}{ Severity of AKI (vs. stage 1 and stage 2) } \\
\hline KDIGO stage 3 & $25.11(1.86-58.05)$ & 0.001 & $17.86(1.20-265.49)$ & $<0.005$ \\
\hline Baseline eGFR & $1.049(0.971-1.010)$ & 0.941 & & \\
\hline Baseline serum creatinine & $1.039(0.737-1.464)$ & 0.829 & & \\
\hline Admission serum creatinine & $1.512(1.094-2.090)$ & 0.012 & & \\
\hline Urine NGAL/Cr & $1.035(1.003-1.067)$ & 0.030 & & \\
\hline Urine KIM-1/Cr & $1.008(0.996-1.021)$ & 0.208 & & \\
\hline \multicolumn{5}{|c|}{ Urine TIMP-2/IGFBP7 (vs. 1st and 2nd quartile group) } \\
\hline 3rd Quartile group & $8.48(0.735-97.77)$ & 0.087 & $5.75(0.67-49.48)$ & 0.011 \\
\hline 4th Quartile group & $79.75(5.19-1,225.37)$ & 0.002 & $44.98(4.40-460.29)$ & 0.001 \\
\hline
\end{tabular}

In the multivariate analysis model, a backward (LR) selection approach was adopted.

OR, odds ratio; CI, confidence interval; DM, diabetes mellitus; HTN, hypertension; CKD, chronic kidney disease; AKI, acute kidney injury; KDIGO, Kidney Disease Improving Global Outcomes; eGFR, estimate glomerular filtration rate; NGAL, neutrophil gelatinase-associated lipocalin; Cr, creatinine; KIM-1, kidney injury molecule 1; TIMP-2, tissue inhibitor of metalloproteinase-2; IGFBP 7 , insulin-like growth factor-binding protein 7.

further examine the predictive performance of urinary TIMP-2 and IGFBP7 for RRT and renal recovery from AKI, we plotted the receiver operating characteristics (ROC) curve using the sensitivity and specificity of urine TIMP-2, IGFBP7. A p value $<0.05$ was considered statistically significant.

\section{RESULTS}

\section{Urine TIMP-2/IGFBP7 at the time of AKI diagnosis could predict adverse outcome}

One hundred and twenty-four patients with a clinical diagnosis of AKI were prospectively enrolled. The mean age was $71.5 \pm 14.3$ years and the mean baseline serum creatinine level was $1.21 \pm 0.45 \mathrm{mg} / \mathrm{dL}$. Seventy-nine patients (63.7\%) were diagnosed as KDIGO stage 2 and 3 se- 
Table 4. Univariate and multivariate analyses for predicting non-recovery

\begin{tabular}{|c|c|c|c|c|}
\hline \multirow{2}{*}{ Variable } & \multicolumn{2}{|c|}{ Univariate analysis } & \multicolumn{2}{|c|}{ Multivariate analysis } \\
\hline & OR $(95 \% \mathrm{CI})$ & $p$ value & OR (95\% CI) & $p$ value \\
\hline Age & $0.552(0.188-1.620)$ & 0.280 & & \\
\hline Sex & $1.230(0.500-3.029)$ & 0.552 & & \\
\hline $\mathrm{DM}$ & $2.427(0.924-6.380)$ & 0.072 & & \\
\hline HTN & $1.177(0.503-2.757)$ & 0.707 & & \\
\hline CKD & $1.622(0.520-5.056)$ & 0.405 & & \\
\hline \multicolumn{5}{|c|}{ Severity of AKI (vs. stage 1 and stage 2) } \\
\hline KDIGO stage 3 & $3.362(1.192-9.482)$ & 0.022 & $2.468(1.063-5.728)$ & 0.035 \\
\hline Baseline eGFR & $3.129(0.976-1.012)$ & 0.067 & & \\
\hline Baseline serum creatinine & $1.058(0.834-1.341)$ & 0.643 & & \\
\hline Admission serum creatinine & $0.894(0.732-1.092)$ & 0.271 & & \\
\hline Urine NGAL/Cr & $1.013(0.977-1.051)$ & 0.480 & & \\
\hline Urine KIM-1/Cr & $1.006(0.996-1.017)$ & 0.229 & & \\
\hline \multicolumn{5}{|c|}{ Urine TIMP-2/IGFBP 7 (vs. 1st and 2nd quartile group) } \\
\hline 3rd Quartile group & $1.652(0.524-5.210)$ & 0.039 & $1.896(0.648-5.549)$ & 0.024 \\
\hline 4th Quartile group & $3.361(1.188-9.505)$ & 0.022 & $3.622(1.358-9.665)$ & 0.010 \\
\hline
\end{tabular}

In the multivariate analysis model, a backward (LR) selection approach was adopted.

OR, odds ratio; CI, confidence interval; DM, diabetes mellitus; HTN, hypertension; CKD, chronic kidney disease; AKI, acute kidney injury; KDIGO, Kidney Disease Improving Global Outcomes; eGFR, estimate glomerular filtration rate; NGAL, neutrophil gelatinase-associated lipocalin; Cr, creatinine; KIM-1, kidney injury molecule 1; TIMP-2, tissue inhibitor of metalloproteinase-2; $\mathrm{IGFBP}_{7}$, insulin-like growth factor-binding protein 7.

vere AKI and 16 out of 124 (12.9\%) received RRT (Table 1 ). We divided these patients into quartiles according to the initial urine TIMP-2/IGFBP7 level $\left(\log _{10}\right.$ transformed, $<1.10,1.10-1.58,1.59-2.11,2.12 \leq$ ) and compared the baseline characteristics as well as the outcomes. Age, sex, comorbidities such as hypertension and diabetes, initial serum creatinine at the time of diagnosis of AKI, or the severity of AKI defined by KDIGO staging were not different according to the quartiles (Table 1). However, the requirement of RRT showed a stepwise increase according to quartiles with the highest being in the fourth quartiles (Table 2).

The in-hospital mortality rate was $5.5 \%$ and there was no difference in the mortality rate among the quartiles. Of 118 survivors, 90 (76.2\%) showed functional recovery at discharge. In the first quantile, $93.5 \%$ of the patients achieved functional recovery, whereas only $54.8 \%$ of the fourth quartile recovered (Table 2). In predicting nonrecovery, both KDIGO stage 3 and the third and fourth quartiles of TIMP-2/IGFBP7 were significant. However, none of these could predict the in-hospital mortality or length of hospital stay (Table 2).

The logistic regression analysis demonstrated that KDIGO stage 3 AKI (odds ratio [OR], 17.86), as well as the third and fourth quartiles of TIMP-2/IGFBP7 (OR, 5.75 and 44.98 , respectively), were found to be independent factors predicting RRT at the time of AKI diagnosis (Table 3). KDIGO stage 3 AKI (OR, 2.468) or the third or fourth quartiles of urinary TIMP-2/IGFBP7 (OR, 1.896 and 3.622, respectively) were also found to be useful in predicting nonrecovery of renal function using logistic regression analysis (Table 4). To minimize the comorbid conditions that can affect urine results, the predictive value of TIMP-2/IGFBP7 was further analyzed in patients without proteinuria $(\geq 300 \mathrm{mg} / \mathrm{dL}$ ) or bilirubinuria as they may interfere with the test. In the subgroup analysis, we found that initial urine TIMP-2/IGFBP7 level still significantly predicted RRT requirement and non-recovery (Supplementary Table 1). 

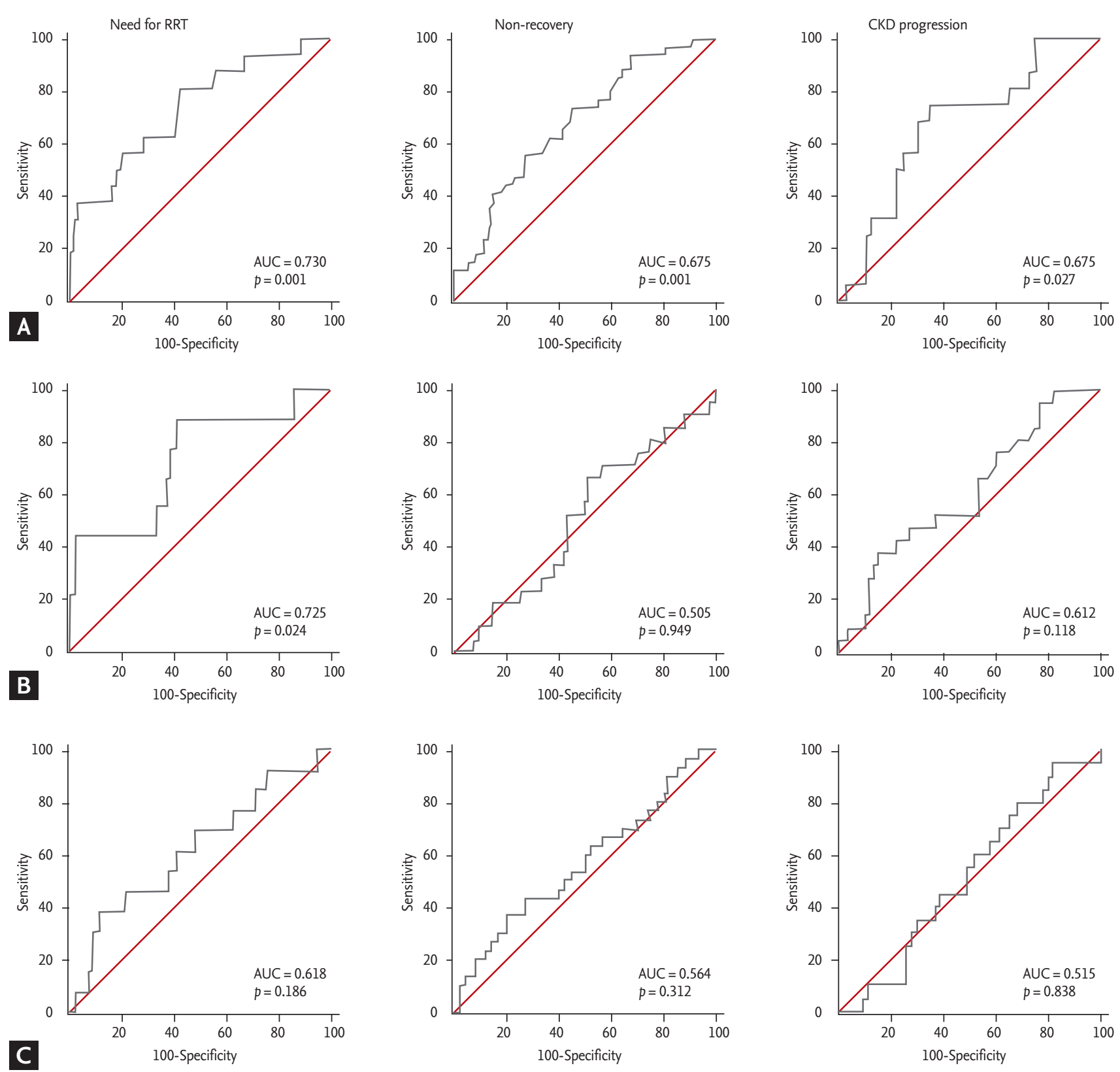

Figure 1. Receiver operating characteristic curve of (A) initial urine tissue inhibitor of metalloproteinase-2/insulin-like growth factor-binding protein 7 (TIMP-2/IGFBP7), (B) urine neutrophil gelatinase-associated lipocalin/creatinine (NGAL/Cr), and (C) urine kidney injury molecule 1 (KIM-1)/Cr levels for prediction of the need for renal replacement therapy (RRT), non-recovery, and new-onset or progressive chronic kidney disease (CKD). AUC, area under the curve.

\section{Prognostic performance of urine TIMP-2/IGFBP7}

The prognostic performance of urinary TIMP-2/IGFBP7 in predicting RRT or functional recovery was determined by calculating the area under the curve (AUC) and compared with that of urinary NGAL and KIM-1, which have been validated as prognostic markers in several studies
(Fig. 1). The urinary TIMP-2/IGFBP7 level at the time of AKI diagnosis showed reasonable prognostic performance for predicting RRT (AUC, $0.730 ; p=0.001$ ), similar to that of urinary NGAL (AUC, $0.725 ; p=0.024$ ) and better than that of urinary KIM-1 (AUC, $0.618 ; p=0.186$ ). At a cutoff value of 1.61, the sensitivity and specificity for pre- 
Table 5. Multivariate analyses for predicting CKD progression

\begin{tabular}{lcc}
\hline Variable & Odds ratio (95\% CI) & p value \\
\hline Urine TIMP-2/IGFBP7 & $2.274(1.021-5.056)$ & 0.044 \\
Discharge IGFBP7 & $1.111(1.011-1.237)$ & 0.045 \\
\hline
\end{tabular}

In the multivariate analysis model, a backward (LR) selection approach was adopted. Other parameters (age, sex, diabetes mellitus, hypertension, severity of acute kidney injury, urine neutrophil gelatinase-associated lipocalin/creatinine [Cr], urine kidney injury molecule 1 [KIM-1]/Cr) except urine TIMP-2/IGFBP 7 , discharge IGFBP 7 , were not displayed. Discharge $\mathrm{IGFBP}_{7}, \mathrm{IGFBP}_{7}$ levels at the time of discharge. CKD, chronic kidney disease; CI, confidence interval; TIMP-2, tissue inhibitor of metalloproteinase-2; IGFBP7, insulin-like growth factor-binding protein 7 .

dicting RRT were $81.2 \%$ and $57.4 \%$, respectively. In addition, the prognostic performance of urinary TIMP-2/ $\mathrm{IGFBP}_{7}$ in predicting functional nonrecovery was only modest (AUC, $0.675 ; p=0.001$ ) but better than that of urinary NGAL (AUC, $0.505 ; p=0.949$ ) and urinary KIM-1 (AUC, $0.564 ; p=0.312$ ). KDIGO staging at the time of biomarker measurement also showed good performance in predicting RRT and nonrecovery, and pairwise comparison of the ROC curve between urinary TIMP-2/IGFBP7 and KDIGO showed no significant differences.

\section{Urine TIMP-2/IGFBP7 can predict new-onset CKD or progression}

Three months after discharge from the hospital, we assessed the development of new-onset CKD or progression of CKD in 90 patients who achieved functional recovery. Although $76.2 \%$ (90 patients of 118 survivors) showed functional recovery at the time of hospital discharge, 10 patients (11.1\%) developed new-onset CKD and 20 patients $(22.2 \%)$ showed more than a $25 \%$ reduction of eGFR, suggesting the progression of CKD at 3 months after discharge. From the logistic regression analysis, the initial urine TIMP-2/IGFBP7 level was also found to be useful in predicting the development of new-onset or progression of CKD (Table 5). The performance of initial urinary TIMP-2/IGFBP 7 in predicting CKD progression was also determined by calculating AUC and compared to that of urinary NGAL and KIM-1. Only urinary TIMP-2/IGFBP7 levels were significant for predicting new-onset or progressive $\mathrm{CKD}$ according to the AUC analysis (Fig. 1). Urine was also collected at dis- charge and analyzed for TIMP-2/IGFBP 7 levels, TIMP2, and $\mathrm{IGFBP}_{7}$, respectively, to determine whether $\mathrm{CKD}$ progression could be predicted. Only the urine IGFBP7 level in this analysis significantly predicted new-onset or progressive CKD (Table 5).

\section{DISCUSSION}

Our results showed that urinary TIMP-2/IGFBP7 on the first day of AKI diagnosis could reliably predict the requirement of RRT, as well as functional recovery. We also demonstrated that the initial TIMP-2/IGFBP7 or $\mathrm{IGFBP}_{7}$ level at the time of discharge could predict new-onset or progressive CKD in patients who achieved short-term functional recovery from AKI.

AKI is not only associated with increased in-hospital mortality but also with long-term adverse outcomes including progression to ESRD or late mortality [1]. In addition, recent studies demonstrated that severe AKI requiring dialysis is even associated with increased risk of stroke, congestive heart failure, or gastrointestinal bleeding [10]. Although observational studies suggested that the duration and severity of AKI could be parameters predicting these adverse outcomes, accurate prediction is not easy in clinical practice $[4,11]$.

Two novel biomarkers, urine TIMP-2 and IGFBP7, were recently discovered and validated in two-stage multicenter studies for their ability to predict the development of KDIGO stage 2 or 3 AKI within 12 hours in high-risk patients [6]. Both of these markers represent Gi cell cycle arrest, and their expression is increased after ischemic or toxic insults. The United States Food and Drug Administration allowed marketing of NephroCheck, which can measure these markers simultaneously, as the first laboratory test to help determine the risk of developing AKI in critically ill patients. A subsequent study also showed that high levels of TIMP-2 and $\mathrm{IGFBP}_{7}$ were associated with death or RRT. However, their predictive value for renal recovery and CKD progression is not well-known [12].

Unlike previous studies that enrolled only high-risk or critically ill patients without clinical evidence of AKI, we collected urine from patients with established AKI on the first day of enrollment. Because the level of biomarker during or after AKI has not been determined to 
predict AKI outcome and it is still unknown for how long TIMP-2/IGFBP7 will increase after AKI, we attempted to test whether these two markers were also useful in predicting the need for RRT, in-hospital mortality, and renal recovery in heterogenous patients with established AKI. As most patients in our study were not critically ill patients admitted to intensive care units, the percentage of patients who required dialysis (12.9\%) and the in-hospital mortality (5.6\%) were relatively low. However, when we divided the patients into the 4 quartiles according to initial urine TIMP-2/IGFBP 7 , the patients in the fourth quartile showed the highest rate of RRT and lowest rate of functional recovery. In multivariate analysis, KDIGO stage 3 and third and fourth quartiles of TIMP-2/ $\mathrm{IGFBP}_{7}$ at the time of AKI diagnosis were found to be useful in predicting dialysis requirements and renal recovery. However, given that staging of AKI usually takes several days following the initial diagnosis, the clinical significance of urine TIMP-2/IGFBP7 in terms of early prediction of dialysis is far greater.

The failure to predict in-hospital mortality was likely the result of a relatively low mortality rate in our cohort (seven out of 125, 5.6\%). The performance of urine TIMP-2/IGFBP7 in predicting RRT requirement was quite good, similar to NGAL, which is another promising biomarker that has been validated in many studies [13]. The cutoff value for TIMP-2/IGFBP7 to predict RRT requirement was 1.61, which was much higher than the level used to predict AKI in critically ill patients who have an increased risk of AKI. The Sapphire study demonstrated that the risk of stage 2 to 3 AKI increased sharply at a value above 0.3 measured using the NephroCheck diagnostic kit [6]. However, a direct comparison is somewhat difficult because our cohort consisted of established AKI patients who would have higher levels of urinary injury biomarkers than patients at high risk of, but not established, AKI. To the best of our knowledge, this is the first report that showed the possibility that TIMP-2/IGFBP 7 in established AKI patients could serve as a useful prognostic marker of RRT requirement. If RRT requirement can be accurately predicted with urine TIMP-2/IGFBP7, combined with several clinical parameters during an earlier time point, it might help clinicians avoid unnecessary fluid resuscitation and diuretic therapy early in the course of AKI.

In this study, because of enrollment of patients with known baseline renal function, we could reliably verify patients with functional renal recovery, which was defined as return of the serum creatinine level to a level $25 \%$ of the baseline level. Among the 118 patients who survived, 90 patients $(76.3 \%)$ showed recovery at the time of discharge, For them, the TIMP-2/IGFBP7 levels on the first day could also predict functional renal recovery with modest performance (AUC, 0.675). Recently, Aregger et al. [14] also demonstrated that urine IGFBP7, collected on the first day of AKI in critically ill patients, could predict renal recovery. By proteomic analysis of urine from early recovery versus late recovery/nonrecovery patients, they identified several candidate markers and found that $\mathrm{IGFBP}_{7}$, followed by NGAL, was useful in predicting not only severity but also renal recovery. However, validation was performed in only 28 patients and 12 controls. Our study, in contrast, analyzed 118 patients who survived from AKI and showed that IGFBP7, combined with TIMP-2, measured on the first day of AKI diagnosis could predict renal recovery, but urinary NGAL could not.

Observations from many epidemiological studies, which indicated that episodes of AKI are the most important factor in the development or progression of $\mathrm{CKD}$, led us to determine renal function 3 months after discharge [15]. Although there were no patients who had a second relapse of AKI during the 3 months period, 10 patients developed new-onset CKD and 20 patients progressed defined as $25 \%$ or more decline of eGFR. We analyzed multiple factors that could predict this later renal outcome and found that the initial TIMP-2/IGFBP7 level could predict new-onset or progressive CKD, even though their renal function showed recovery at the time of discharge. In addition, we also found that IGFBP7 in urine, obtained at the time of hospital discharge, could also predict new-onset or progressive CKD. This shows that even after the achievement of functional recovery, patients with persistently elevated urinary $\mathrm{IGFBP}_{7}$ are still at high risk of incident or progressive CKD. The observation that prolonged cell cycle arrest is associated with maladaptive repair and progressive fibrosis in animal models of AKI to CKD transition could also support our findings [16].

Despite several meaningful findings, our study has several limitations. First, it is a single-center study, inherently associated with a lack of generalizability to 


\section{KJIM}

broader populations. Second, because our study measured the biomarker at different time points after AKI development, it is not easy to provide a standardized cut off value to predict the AKI outcome, and it may not be appropriate to apply the cut-off value to AKI patients in different situations. Third, we measured TIMP-2 and $\mathrm{IGFBP}_{7}$ separately using ELISA kits rather than using commercial NephroCheck because it is unavailable outside the USA. Therefore, it makes a direct comparison difficult and it may not be appropriate to apply non-standardized ELISA results to clinical practice. Lastly, the relatively lower severity of illness or AKI due to enrollment of established AKI patients from the general ward make it difficult to apply these data to the condition of critically ill patients. However, the enrolling of AKI patients with known baseline renal function leading to more accurate verification of renal recovery is a strong point of our study. In addition, testing the biomarkers in the urine sample collected after functional recovery indicated the possibility of using these markers for assessment of incident or progressive CKD, which is of interest.

In conclusion, our study showed the possibility that requirement of RRT or renal recovery could be predicted on the first day of AKI by measuring urine TIMP2/IGFBP7 levels before AKI staging by measuring daily serum creatinine levels or urine output. Although this might imply that early decisions made on the basis of these markers regarding RRT issues (including timing, mode, or other supportive measures) might improve patient outcomes, it obviously needs further thorough investigation in a larger number of patients. We also demonstrated that even after renal recovery, a substantial percentage of patients was still at high risk of incident or progressive CKD, including those who did not receive RRT. The urine TIMP-2/IGFBP7 at the time of AKI diagnosis or increased urine IGFBP 7 even after functional recovery might be useful in predicting the long-term renal outcome.

\section{KEY MESSAGE}

1. Initial urinary tissue inhibitor of metalloproteinase-2/insulin-like growth factor-binding protein 7 (TIMP-2/IGFBP7) can predict the need for renal replacement therapy and nonrecovery of renal function in acute kidney injury patients.

2. Initial urinary TIMP-2/IGFBP 7 or urinary $\mathrm{IGFBP}_{7}$ at the time of discharge can predict new-onset or progressive chronic kidney disease following acute kidney injury.

\section{Conflict of interest}

No potential conflict of interest relevant to this article was reported.

\section{REFERENCES}

1. Lafrance JP, Miller DR. Acute kidney injury associates with increased long-term mortality. J Am Soc Nephrol 2010;21:345-352.

2. Doyle JF, Forni LG. Acute kidney injury: short-term and long-term effects. Crit Care 2016;20:188.

3. Chawla LS, Eggers PW, Star RA, Kimmel PL. Acute kidney injury and chronic kidney disease as interconnected syndromes. N Engl J Med 2014;371:58-66.

4. Leung KC, Tonelli M, James MT. Chronic kidney disease following acute kidney injury-risk and outcomes. Nat Rev Nephrol 2013;9:77-85.

5. Rifkin DE, Coca SG, Kalantar-Zadeh K. Does AKI truly lead to CKD? J Am Soc Nephrol 2012;23:979-984.

6. Kashani K, Al-Khafaji A, Ardiles T, et al. Discovery and validation of cell cycle arrest biomarkers in human acute kidney injury. Crit Care 2013;17:R25.

7. Canaud G, Bonventre JV. Cell cycle arrest and the evolution of chronic kidney disease from acute kidney injury. Nephrol Dial Transplant 2015;30:575-583.

8. Ronco C. Cell cycle arrest biomarkers: new weapons for a new battle. Blood Purif 2014;38:I-III.

9. Levey AS, Stevens LA, Schmid CH, et al. A new equation to estimate glomerular filtration rate. Ann Intern Med 2009;150:604-612.

10. Wu VC, Wu CH, Huang TM, et al. Long-term risk of coronary events after AKI. J Am Soc Nephrol 2014;25:595-605.

11. Chawla LS, Kimmel PL. Acute kidney injury and chronic kidney disease: an integrated clinical syndrome. Kidney Int 2012;82:516-524.

12. Koyner JL, Shaw AD, Chawla LS, et al. Tissue inhibitor 
metalloproteinase-2 (TIMP-2) · IGF-binding protein-7 (IG$\mathrm{FBP}_{7}$ ) levels are associated with adverse long-term outcomes in patients with AKI. J Am Soc Nephrol 2015;26:1747-1754.

13. Cruz DN, de Cal M, Garzotto F, et al. Plasma neutrophil gelatinase-associated lipocalin is an early biomarker for acute kidney injury in an adult ICU population. Intensive Care Med 2010;36:444-451.

14. Aregger F, Uehlinger DE, Witowski J, et al. Identification of IGFBP-7 by urinary proteomics as a novel prog- nostic marker in early acute kidney injury. Kidney Int 2014;85:909-919.

15. Sawhney S, Mitchell M, Marks A, Fluck N, Black C. Longterm prognosis after acute kidney injury (AKI): what is the role of baseline kidney function and recovery? A systematic review. BMJ Open 2015;5:e006497.

16. Lim SY, Ko YS, Lee HY, et al. The impact of preexisting chronic kidney disease on the severity and recovery of acute kidney injury. Nephron 2018;139:254-268. 
Supplementary Table 1. Univariate analyses for predicting renal outcomes in patients without proteinuria ( $\geq 300 \mathrm{mg} / \mathrm{dL}) \mathrm{or}$ bilirubinuria

Variable

